\title{
ON NONSTRONGLY REGULAR MATRICES
}

\author{
J. BAZINET AND J. A. SIDDIQI ${ }^{1}$
}

AbStRaCt. Using the Rudin-Shapiro sequence, the existence of a regular but not strongly regular positive matrix that sums $\{\exp (2 \pi i k t)\}$ to 0 for all $t \in(0,1)$ is established. As a corollary it is shown that there exist matrices that sum all almost periodic sequences without possessing the Borel property and vice versa.

1. A matrix $A=\left(a_{n, k}\right)$ of real or complex numbers is called strongly regular if it is regular and

$$
\lim _{n \rightarrow \infty} \sum_{k=0}^{\infty}\left|a_{n, k}-a_{n, k+1}\right|=0 .
$$

Such matrices possess many interesting properties. Thus a matrix is translative if and only if it is strongly regular (cf. Petersen [5, p. 21]). Lorentz [3] has shown that a matrix sums every almost convergent sequence to its almost convergence limit if and only if it is strongly regular.

If $A=\left(a_{n, k}\right)$ is strongly regular, then it necessarily satisfies the conditions

$$
\lim _{n \rightarrow \infty} \sum_{k=0}^{\infty} a_{n, k} \exp (2 \pi i k t)=0
$$

and

$$
\lim _{n \rightarrow \infty} \sum_{k=0}^{\infty}\left|a_{n, k}\right| \exp (2 \pi i k t)=0
$$

for all $t \in(0,1)$ as a simple Abel's transformation shows. The question naturally arises whether there exist regular matrices satisfying (2) and (3) that are not strongly regular. A positive answer to this question becomes much more significant if we observe that the Fourier-effective matrices

Received by the editors October 12, 1970 and, in revised form, December 9, 1970. AMS 1970 subject classifications. Primary 40D25, 40C99.

Key words and phrases. Regular, strongly regular, almost convergence, Fouriereffective, Borel property, almost periodic.

${ }^{1}$ The research was supported by the National Research Council of Canada grant A-4057 to the second author.

(c) American Mathematical Society 1972 
considered by Hille and Tamarkin [2], Nikol'skiǐ [4] and Sz.-Nagy [8] have to satisfy (2) and (3) though perhaps not (1).

2. In this paper we construct a nonstrongly regular positive regular matrix satisfying (2) and (3) possessing, in addition, the Borel property of summing almost all sequences of 0 's and 1's to the value $\frac{1}{2}$. The construction is based on the use of the coefficients of the Rudin-Shapiro polynomial as given in Rudin [6]. These are defined as follows.

We set $P_{0}(x)=Q_{0}(x)=x$ and define $P_{k}$ and $Q_{k}$ inductively by

$$
\begin{aligned}
& P_{k+1}(x)=P_{k}(x)+x^{2^{k}} Q_{k}(x) \\
& Q_{k+1}(x)=P_{k}(x)-x^{2^{k}} Q_{k}(x)
\end{aligned} \quad(k=0,1,2, \cdots) .
$$

Clearly $P_{1}(x)=x+x^{2}$ and $Q_{1}(x)=x-x^{2}$. We observe that $P_{k}$ is a polynomial of degree $2^{k}$ and that $P_{k}$ is a partial sum of $P_{k+1}$. Hence we can define a sequence $\left\{\varepsilon_{n}\right\}$ by setting $\varepsilon_{n}$ equal to the $n$th coefficient of $P_{k}$, where $2^{k}>n$. Clearly $\varepsilon_{n}=1$ or -1 . It has been shown by Rudin [6] that

$$
\left|\sum_{n=1}^{N} \varepsilon_{n} e^{i n \theta}\right|<5 \sqrt{ } N \text { for } 0 \leqq \theta \leqq 2 \pi, N=1,2, \cdots \text {. }
$$

We assert that for all $k=1,2, \cdots$,

and

$$
\begin{aligned}
P_{k+1}= & P_{1}+x^{2} Q_{1}+\delta_{4} x^{4} P_{1}+\delta_{6} x^{6} Q_{1}+\cdots \\
& +\delta_{2^{k+1}-4} x^{2^{k+1}-4} P_{1}+\delta_{2^{k+1}-2} x^{2^{k+1}-2} Q_{1}
\end{aligned}
$$

$$
\begin{aligned}
Q_{k+1}= & P_{1}+x^{2} Q_{1}+\delta_{4}^{(k)} x^{4} P_{1}+\delta_{6}^{(k)} x^{6} Q_{1}+\cdots \\
& +\delta_{2^{k+1}-4}^{(k)} x^{2^{k+1}-4} P_{1}+\delta_{2^{k+1}-2}^{(k)} x^{2^{k+1}-2} Q_{1}
\end{aligned}
$$

where $\delta_{2 k}$ and $\delta_{2 k}^{(k)}$ are either +1 or -1 . The assertion is true for $k=1$. If we assume that it is true for $k-1$, then using (4) we get

$$
\begin{aligned}
P_{k+1}= & P_{k}+x^{2^{k}} Q_{k} \\
= & \left(P_{1}+x^{2} Q_{1}+\cdots+\delta_{2_{-4}^{k}} x^{2^{k}-4} P_{1}+\delta_{2^{k}-2} x^{2^{k}-2} Q_{1}\right) \\
& +x^{2^{k}}\left(P_{1}+x^{2} Q_{1}+\cdots+\delta_{2^{k}-4}^{(k-1)} x^{2^{k}-4} P_{1}+\delta_{2^{k}-2}^{(k-1)} x^{2^{k}-2} Q_{1}\right) \\
= & P_{1}+x^{2} Q_{1}+\cdots+\delta_{2^{k+1}-4} x^{2^{k+1}-4} P_{1}+\delta_{2^{k+1}-2} x^{2^{k+1}-2} Q_{1}
\end{aligned}
$$

and similarly for $Q_{k+1}$.

We therefore have

$$
P_{k+1}(x)=\sum_{r=0}^{2^{k-1}}\left\{\delta_{4 r} x^{4 r} P_{1}(x)+\delta_{4 r+2} x^{4 r+2} Q_{1}(x)\right\}
$$

where $\delta_{0}=\delta_{2}=1$. The general term in the above summation can be only 
one of the following forms:

$$
\begin{aligned}
x^{4 r} P_{1}(x)+x^{4 r+2} Q_{1}(x) & =x^{4 r+1}+x^{4 r+2}+x^{4 r+3}-x^{4 r+4}, \\
x^{4 r} P_{1}(x)-x^{4 r+2} Q_{1}(x) & =x^{4 r+1}+x^{4 r+2}-x^{4 r+3}+x^{4 r+4} \\
-x^{4 r} P_{1}(x)+x^{4 r+2} Q_{1}(x) & =-x^{4 r+1}-x^{4 r+2}+x^{4 r+3}-x^{4 r+4} \\
-x^{4 r} P_{1}(x)-x^{4 r+2} Q_{1}(x) & =-x^{4 r+1}-x^{4 r+2}-x^{4 r+3}+x^{4 r+4}
\end{aligned}
$$

it follows that the set $\left\{\varepsilon_{4 n+1}, \varepsilon_{4 n+2}, \varepsilon_{4 n+3}, \varepsilon_{4 n+4}\right\}$ consists of either three +1 's and one -1 or three -1 's and one +1 .

Consider the matrix $A=\left(a_{n, k}\right)$ defined as follows:

$$
\begin{aligned}
a_{n, k} & =1 / 2 n & & \text { for } 1 \leqq k \leqq n, \\
& =\left(\varepsilon_{k-n}+1\right) / 2 n & & \text { for } n+1 \leqq k \leqq 2 n, \\
& =0 & & \text { for } k>2 n .
\end{aligned}
$$

$A$ is regular since $\sum_{k=1}^{\infty} a_{n, k}=1+(1 / 2 n) \sum_{1}^{n} \varepsilon_{k}$ where the second term on the right tends to zero as $n \rightarrow \infty$ by (5) and other conditions are easily seen to be satisfied. Since

$$
\begin{aligned}
\sum_{k=1}^{\infty}\left|a_{n, k}\right| \exp (2 \pi i k t) & =\sum_{k=1}^{\infty} a_{n, k} \exp (2 \pi i k t) \\
= & \frac{1}{2 n}\left[\sum_{k=1}^{2 n} \exp (2 \pi i k t)+\sum_{k=1}^{n} \varepsilon_{k} \exp \{2 \pi i(k+n) t\}\right],
\end{aligned}
$$

it follows again by (5) that $A$ satisfies (2) and (3). Finally, since

$$
\begin{aligned}
\sum_{k=1}^{\infty} \mid a_{n, k} & -a_{n, k+1} \mid \\
& \geqq \frac{1}{2 n} \sum_{k=0}^{[n / 4]-1}\left\{\left|\varepsilon_{4 k+1}-\varepsilon_{4 k+2}\right|+\left|\varepsilon_{4 k+2}-\varepsilon_{4 k+3}\right|+\left|\varepsilon_{4 k+3}-\varepsilon_{4 k+4}\right|\right\} \\
& \geqq(1 / 2 n) 2\{[n / 4]-1\},
\end{aligned}
$$

$A$ does not satisfy (1).

We now show that $A$ possesses the Borel property. This follows readily from a result due to Hill [1, p. 405] which asserts that $A$ has the Borel property if $\sum_{n=1}^{\infty} A_{n}^{2}<\infty$ where $A_{n}=\sum_{k=1}^{\infty}\left|a_{n, k}\right|^{2}$ since in our case $A_{n} \leqq 3 / 4 n$.

3. In a recent paper [7], one of the authors has proved that a matrix $A=\left(a_{n, k}\right)$ sums every almost periodic sequence if and only if (1) $\|A\|=$ $\sup _{n \geqq 0} \sum_{k=0}^{\infty}\left|a_{n, k}\right|<\infty$, and (2) $\lim _{n \rightarrow \infty} \sum_{k=0}^{\infty} a_{n, k} \exp (2 \pi i k t)$ exists for all $t \in(0,1)$. He calls such matrices almost periodic. The matrix $A$ constructed in $\S 2$ is clearly an almost periodic matrix that possesses the Borel property. However an almost periodic matrix need not necessarily possess the Borel property. If we use the notation $a(n, k)$ as alternative 
to $a_{n, k}$ and define

$$
a\left\{\left(m^{2}+i-1\right),\left((m-1)^{-2}+j-2\right)\right\} \equiv[\log m]^{-1}
$$

for $j=i+1, i+2, \cdots, i+[\log m] ; i=1,2, \cdots, 2 m+1 ; m=3,4,5, \cdots$, wherein, as usual, $[\log m]$ means the greatest integer in $\log m$, and let $a_{n, k} \equiv 0$ otherwise. Then, as shown in [1], $A=\left(a_{n, k}\right)$ is a matrix of nonnegative entries which does not possess the Borel property but is almost periodic since, for $t \not \equiv 0(\bmod 2 \pi)$,

$$
\begin{aligned}
& {[\log m]^{-1} \sum_{j=k+1}^{k+[\log m]} \exp \left(\left\{(m-1)^{2}+j-2\right\} i t\right)} \\
& \quad=[\log m]^{-1} \exp \left(\left\{(m-1)^{2}+k-1\right\} i t\right) \frac{1-\exp (i[\log m] t)}{1-\exp (i t)}
\end{aligned}
$$

tends to 0 as $m \rightarrow \infty$ and, for $t \equiv 0(\bmod 2 \pi)$,

$$
[\log m]^{-1} \sum_{j=k+1}^{k+[\log m]} \exp \left(\left\{(m-1)^{2}+j-2\right\} i t\right)=1 .
$$

Also there exist matrices possessing the Borel property without being necessarily almost periodic. The matrix $A=\left(a_{n, k}\right)$ with $a_{n, 0}=0$, $a_{n, k}=1 / n$ for $1 \leqq k \leqq n$ and $a_{n, n+k}=(-1)^{n+k-1} /(n+k)$ for $k>n$ is such that (1) $\lim _{n \rightarrow \infty} a_{n, k}=0$, (2) $\lim _{n \rightarrow \infty} \sum_{k=0}^{\infty} a_{n, k}=1$ but (3) $\|A\|=$ $\sup _{n \geqq 0} \sum_{k=0}^{\infty}\left|a_{n, k}\right|=+\infty$, so that $A$ is not almost periodic. But $A$ has the Borel property since

$$
A_{n}=\sum_{k=0}^{\infty}\left|a_{n, k}\right|^{2}=\frac{1}{n}+\sum_{n+1}^{\infty} \frac{1}{k^{2}}=O\left(\frac{1}{n}\right)
$$

so that $\sum_{n=1}^{\infty} A_{n}^{2}<\infty$ and the above quoted criterion of Hill is applicable.

\section{REFERENCES}

1. J. D. Hill, The Borel property of summability methods, Pacific J. Math. 1 (1951), 399-409. MR 13, 340.

2. E. Hille and J. D. Tamarkin, On the summability of Fourier series. I, Trans. Amer. Math. Soc. 34 (1932), 757-783.

3. G. G. Lorentz, A contribution to the theory of divergent sequences, Acta Math. 80 (1948), 167-190. MR 10, 367.

4. S. M. Nikol'skiī, On linear methods of summation of Fourier series, Izv. Akad. Nauk SSSR Ser. Mat. 12 (1948), 259-278. (Russian) MR 10, 247.

5. G. M. Petersen, Regular matrix transformations, McGraw-Hill, New York, 1966. MR 37 \#642.

6. W. Rudin, Some theorems on Fourier coefficients, Proc. Amer. Math. Soc. 10 (1959), 855-859. MR 22 \#6979. 
7. J. A. Siddiqi, Infinite matrices summing every almost periodic sequence, Pacific J. Math. 39 (1971), 235-252.

8. B. Sz.-Nagy, Méthodes de sommation des séries de Fourier. I, Acta Sci. Math. (Szeged) 12 (1950), 204-210. MR 11, 656.

Department of Mathematics, University of Sherbrooke, Sherbrooke, Quebec, CANADA (Current address of both authors)

Summer Research Institute of the Canadian Mathematical Congress, UniverSity of British Columbia, Vancouver, British Columbia, Canada 